University of Nebraska - Lincoln

DigitalCommons@University of Nebraska - Lincoln

Faculty Publications from the Department of Engineering Mechanics

2008

\title{
Simultaneous birefringence, small- and wide-angle X-ray scattering to detect precursors and characterize morphology development during flow-induced crystallization of polymers
}

\author{
Lucia Fernandez-Ballester \\ University of Nebraska - Lincoln, lucia.fernandez@unl.edu \\ Tim Gough \\ University of Bradford (UK) \\ Florian Meneau \\ Netherlands Organization for Scientific Research (NWO) \\ Wim Bras \\ Netherlands Organization for Scientific Research (NWO) \\ Fernando Ania \\ Instituto de Estructura de la Materia, Madrid, Spain \\ See next page for additional authors \\ Follow this and additional works at: https://digitalcommons.unl.edu/engineeringmechanicsfacpub \\ Part of the Mechanical Engineering Commons
}

Fernandez-Ballester, Lucia; Gough, Tim; Meneau, Florian; Bras, Wim; Ania, Fernando; Balta-Calleja, Francisco Jose; and Kornfield, Julia A., "Simultaneous birefringence, small- and wide-angle X-ray scattering to detect precursors and characterize morphology development during flow-induced crystallization of polymers" (2008). Faculty Publications from the Department of Engineering Mechanics. 66.

https://digitalcommons.unl.edu/engineeringmechanicsfacpub/66

This Article is brought to you for free and open access by the Mechanical \& Materials Engineering, Department of at DigitalCommons@University of Nebraska - Lincoln. It has been accepted for inclusion in Faculty Publications from the Department of Engineering Mechanics by an authorized administrator of DigitalCommons@University of Nebraska - Lincoln. 


\section{Authors}

Lucia Fernandez-Ballester, Tim Gough, Florian Meneau, Wim Bras, Fernando Ania, Francisco Jose BaltaCalleja, and Julia A. Kornfield 
Journal of

\section{Synchrotron}

\section{Radiation}

ISSN 0909-0495

Received 30 April 2007

Accepted 23 January 2008
(C) 2008 International Union of Crystallography Printed in Singapore - all rights reserved

\section{Simultaneous birefringence, small- and wide-angle $\mathrm{X}$-ray scattering to detect precursors and characterize morphology development during flow-induced crystallization of polymers}

\author{
Lucia Fernandez-Ballester, ${ }^{\mathrm{a}} \neq$ Tim Gough, ${ }^{\mathrm{b}}$ Florian Meneau, ${ }^{\mathrm{c}} \boldsymbol{T}$ Wim Bras, ${ }^{\mathrm{c}}$ \\ Fernando Ania, ${ }^{d}$ Francisco Jose Balta-Calleja $^{d}$ and Julia A. Kornfield ${ }^{a *}$ \\ a Division of Chemistry and Chemical Engineering, California Institute of Technology, Pasadena, \\ CA 91125, USA, ${ }^{\mathbf{b}}$ University of Bradford, Bradford BD7 1DP, UK, ${ }^{\mathbf{c}}$ Netherlands Organization for \\ Scientific Research (NWO), DUBBLE at ESRF, BP 220, F-38053 Grenoble CEDEX, France, and \\ dInstituto de Estructura de la Materia, CSIC, Serrano 119, 28006 Madrid, Spain. \\ E-mail: jak@cheme.caltech.edu
}

\begin{abstract}
An experimental configuration that combines the powerful capabilities of a short-term shearing apparatus with simultaneous optical and X-ray scattering techniques is demonstrated, connecting the earliest events that occur during shear-induced crystallization of a polymer melt with the subsequent kinetics and morphology development. Oriented precursors are at the heart of the great effects that flow can produce on polymer crystallization (strongly enhanced kinetics and formation of highly oriented crystallites), and their creation is highly dependent on material properties and the level of stress applied. The sensitivity of rheo-optics enables the detection of these dilute shear-induced precursors as they form during flow, before X-ray techniques are able to reveal them. Then, as crystallization occurs from these precursors, X-ray scattering allows detailed quantification of the characteristics and kinetics of growth of the crystallites nucleated by the flow-induced precursors. This simultaneous combination of techniques allows unambiguous correlation between the early events that occur during shear and the evolution of crystallization after flow has stopped, eliminating uncertainties that result from the extreme sensitivity of flow-induced crystallization to small changes in the imposed stress and the material. Experimental data on a bimodal blend of isotactic polypropylenes are presented.
\end{abstract}

Keywords: simultaneous; optical; SAXS; WAXD; flow-induced crystallization; polymer; birefringence.

\section{Introduction}

It is well known that flows applied during polymer processing can significantly alter the kinetics of crystallization and structure development in semicrystalline polymers; therefore, flow and thermal history profoundly impact on final material properties (Haas \& Maxwell, 1969; Andersen \& Carr, 1978; Fujiyama et al., 1988; Kantz et al., 1972; Trotignon \& Verdu, 1987). Study of flow-induced crystallization of polymers poses daunting scientific challenges because of its highly non-linear nature. For example, small changes in the stress imposed on a polymer melt can produce abrupt transitions in kinetics and

$¥$ Current address: Netherlands Organization for Scientific Research (NWO), DUBBLE at ESRF, BP 220, F-38053 Grenoble CEDEX, France.

- Current address: Synchrotron SOLEIL, L'Orme des Merisiers, BP 48, St Aubin, 91192 Gif sur Yvette, France. morphology (Kumaraswamy, Issaian \& Kornfield, 1999). In addition, flow-induced crystallization exhibits extreme sensitivity to material composition (e.g. molar mass distribution) (Vleeshouwers \& Meijer, 1996; Seki et al., 2002). Structure evolution during and after flow involves length scales from $\sim 1 \AA$ to $\sim 10 \mu \mathrm{m}$ and time scales from under $100 \mathrm{~ms}$ to more than $10^{4} \mathrm{~s}$. Especially for events occurring at the fastest time scales, comparisons between results from different techniques (separate instruments) are difficult to synchronize. Thus, it is beneficial to perform simultaneous measurements with complementary experimental techniques, ensuring in this way that the sample and imposed conditions are truly identical (Bras \& Ryan, 1998).

The application of simultaneous X-ray and optical measurements has been well established for quiescent crystallization (Wutz et al., 1995). Overcoming the challenge of 
simultaneously incorporating these methods into flow-induced crystallization processes is the subject of the present paper. Simultaneous X-ray and optical techniques have been applied before in fiber spinning experiments (Ran et al., 2003; Chu \& Hsiao, 2001). Here, we focus on an instrument that imposes well defined stress and thermal histories which places additional constraints on the configuration of the X-ray and optical beams. In our case, the sample is confined to a high-aspectratio channel, which necessitates that light and X-rays propagate along the same axis. Therefore, we combine an approach described previously for studying liquid crystals using simultaneous X-ray and birefringence measurements (Gleeson, 1995) with the short-term shearing strategy that has proved to be a powerful tool for understanding flow-induced crystallization (Liedauer et al., 1993; Vleeshouwers \& Meijer, 1996; Kumaraswamy, Verma \& Kornfield, 1999; Pogodina et al., 1999; Koscher \& Fulchiron, 2002; Somani et al., 2001; Devaux et al., 2004; Azzurri \& Alfonso, 2005; Baert \& Van Puyvelde, 2006; Langouche, 2006; Heeley et al., 2006).

The short-term shearing apparatus (Liedauer et al., 1993; Kumaraswamy, Verma \& Kornfield, 1999) is capable of accessing a high stress regime (which induces highly oriented crystallization) under well defined flow conditions (level of shear stress and duration of shear pulse). In addition, it allows independent control of the temperature history imposed on the polymer; a typical experiment involves raising the temperature above the equilibrium melting point to erase the flow history, cooling down to a shearing temperature where flow is imposed under isothermal conditions, and then either monitoring development of crystallization at the same temperature or under a non-isothermal temperature program. A range of structural probes has been utilized with the shortterm shearing method such as birefringence, turbidity, smallangle X-ray scattering (SAXS), wide-angle X-ray diffraction (WAXD) and optical microscopy (Liedauer et al., 1993; Kumaraswamy, Verma \& Kornfield, 1999; Pogodina et al., 1999; Koscher \& Fulchiron, 2002; Somani et al., 2001; Devaux et al., 2004; Azzurri \& Alfonso, 2005; Baert \& Van Puyvelde, 2006; Langouche, 2006; Heeley et al., 2006). These capabilities have previously allowed isolation of the effects of stress, shearing time and shearing temperature; for example, studying the influence of shearing temperature revealed a kinetic pathway in oriented flow-induced crystallization in isotactic polypropylene (iPP) (Kumaraswamy et al., 2002).

Oriented precursors mediate the enormous effects of flow on polymer crystallization. If the flow conditions are strong enough, thread-like precursors form that increase the kinetics of crystallization by orders of magnitude and nucleate highly oriented crystallites, thus dramatically changing the final morphology. In spite of their importance, the mechanism of creation of thread-like precursors and their dependence on temperature, flow conditions and material properties remains elusive. One reason is that it is difficult to directly detect these precursors. An unusual upturn in the birefringence during flow (Kumaraswamy, Issaian \& Kornfield, 1999; Kumaraswamy et al., 2002) has been shown to be the characteristic signature for the creation of these highly oriented precursors; however,
$\mathrm{X}$-ray scattering techniques have so far been unable to detect the bare precursors, i.e. while they are not yet decorated by oriented crystallites grown on them.

To obtain a better understanding of the fundamental processes that govern flow-induced crystallization, there is a need to connect the events that occur during flow, which are detectable by birefringence but not by SAXS and WAXD, with the subsequent morphology development that can be quantified by X-ray scattering techniques. After cessation of flow, the growth of birefringence is limited to the time range prior to development of substantial turbidity, and interpretation depends on independent measurements (e.g. ratio of parent-to-daughter crystallites in isotactic polypropylene). Synchrotron X-ray scattering techniques are able to provide quantitative information about the crystallization kinetics, degree of crystallinity, orientation distribution of the crystallites, and long period. The present paper aims at achieving the correlation between the events that occur during flow with the progress of crystallization after flow has stopped.

\section{Instrumentation}

\subsection{Rheo-optical apparatus to control flow and thermal history}

The instrument used was based on the short-term shearing apparatus developed by Kumaraswamy (Kumaraswamy, Verma \& Kornfield, 1999), with dimensions modified to fit the beamline configuration. The flow cartridge houses a slit channel geometry with a 1:10 ratio. Two windows are mounted flush on the channel and allow the beam to propagate through the sample in the velocity gradient direction. A conical aperture at the exit of the flow cell allows recording of WAXD up to an angle of $35^{\circ}$ (Fig. 1c). To obtain simultaneous optical and X-ray scattering measurements, the laser and $\mathrm{X}$-ray beams must be collinear and the windows must be transparent to both visible light and $\mathrm{X}$-rays without introducing an excessive deterioration in the data quality. Therefore, diamond windows were used here, in contrast to prior work using quartz windows for optical measurements and beryllium windows for X-ray scattering. Diamond is transparent for $\mathrm{HeNe}$ laser light and it exhibits low X-ray scattering and absorbance. In addition, the mechanical properties of diamond allow it to withstand without deformation the maximum pressures generated inside the flow channel $(\sim 7 \times$ $10^{4} \mathrm{~N} \mathrm{~m}^{-2}$ ). Windows of diamond type $2 \mathrm{~A}$ of dimensions $4 \mathrm{~mm}$ diameter $\times 0.5 \mathrm{~mm}$ were obtained from Harris International and glued to the flow cell with epoxy (353ND from Epotek). Care was taken that the diamond surface was flush with the stainless steel in order to avoid distortion of the flow profile. The clear aperture in the stainless steel cell has a diameter of $1.90 \mathrm{~mm}$.

\subsection{X-ray configuration}

Measurements were performed at the BM26b beamline (DUBBLE) of the European Synchrotron Radiation Facility, Grenoble, France (Bras et al., 2003). The wavelength used was 
(a)

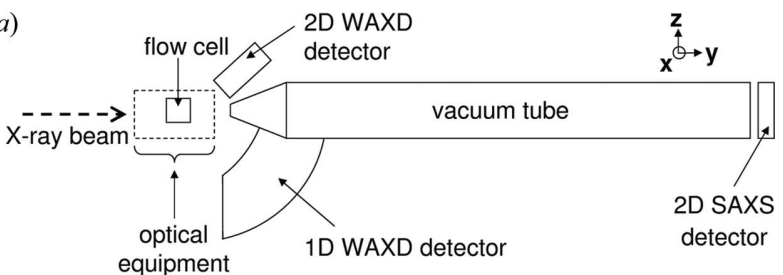

(b)

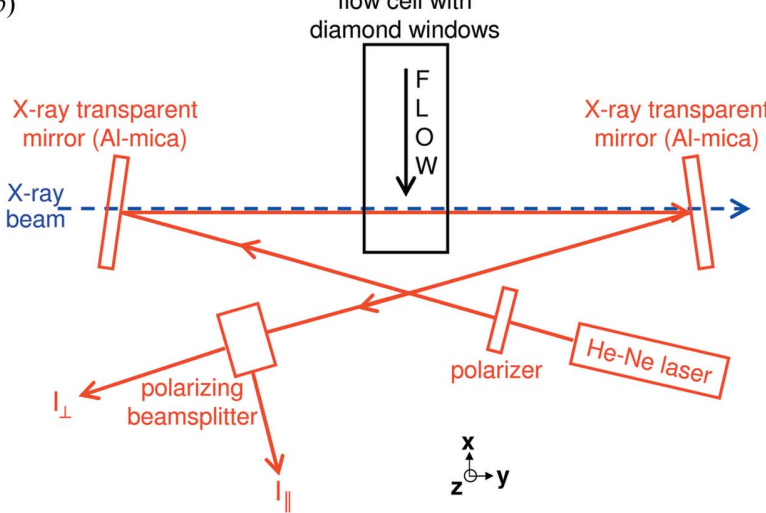

(c)

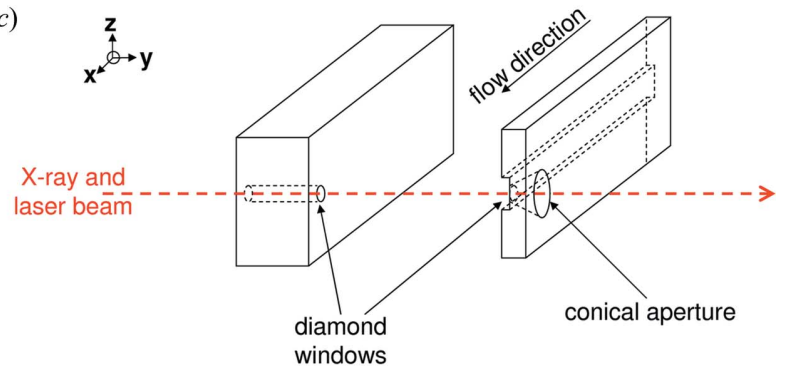

Figure 1

(a) Side view of the experimental configuration showing the relative positions of the flow cell, the optical instrumentation, the WAXD and SAXS detectors and the evacuated tube. The flow direction comes out of the page. (b) Details of the optical instrumentation located in the dashed rectangle in $(a)$ as viewed from the top, indicating the relative position with respect to the flow cell and the X-ray beam. (c) Details of the flow cell composed of two stainless steel pieces that are bolted together, with diamond windows mounted as shown. The incoming light enters a cylindrical aperture and exits through a conical aperture to allow passage of the WAXD pattern up to $35^{\circ}$.

$1.205 \AA$. We performed simultaneous two-dimensional smallangle X-ray scattering (2D SAXS) with one-dimensional wide-angle X-ray diffraction (1DWAXD) (Fig. 1a). In this set-up it is also possible to acquire a sector of 2D WAXD by suitable placement of a CCD camera at an oblique angle with respect to the X-ray beam. A two-dimensional gas-filled multiwired detector was placed $6.7 \mathrm{~m}$ from the sample to collect the SAXS patterns. An evacuated tube was placed between the sample and the detector to minimize air scattering. 1D WAXD was acquired with a curved microstrip gas chamber detector developed at DUBBLE (Zhukov et al., 1997; Dolbnya et al., 2002) that has excellent sensitivity and was placed at the location of the expected equatorial reflections.

\subsection{Optical configuration}

The light source, polarizing optics and detectors were placed such that they did not intersect the path of the X-ray beam (Fig. 1b). A thin aluminium-coated mica sheet was used as a semi-transparent mirror for guiding the laser beam collinear to the X-ray beam. Immediately after the sample cell, a second Al-mica mirror was used to deflect the optical beam to the detection layout. The optical train started with a $17 \mathrm{~mW}$ $\mathrm{HeNe}$ laser $(\lambda=632.8 \mathrm{~nm})$ as the source and a polarizer at $45^{\circ}$ with respect to the flow direction. The outgoing beams $\left(I_{\|}\right.$and $I_{\perp}$ ) were directed by silver mirrors to photodiode detectors for monitoring their intensity.

The mica mirrors consisted of $25 \mu \mathrm{m}$-thick mica sheets coated with $\mathrm{Al}$ on both sides (to avoid bending). The $\mathrm{Al}$ thin films were placed by sputter deposition and had a thickness of $20 \mathrm{~nm}$. These Al-mica mirrors are virtually transparent to $\mathrm{X}$-rays and semi-transparent to $\mathrm{HeNe}$ laser light. The mica sheets were mounted on aluminium holders taking care not to impose stress or deformation upon them. The holder after the sample cell was designed in such a way that it would not interfere with the equatorial WAXD pattern.

\subsection{Experimental procedure}

Operation of the short-term shearing apparatus is controlled by a Labview program, which also records the optical signals and the pressure trace. In turn, the SAXS/ WAXD acquisition program triggers the operation of the Labview program. This ensures accurate synchronization between the shearing pulse, optical and X-ray scattering data.

An example of the experimental protocol for a flowinduced crystallization experiment is described below. Specified temperatures were chosen according to the polymer under investigation. The experimental procedure was as follows (Fig. 2): the flow cell was filled at a low pressure

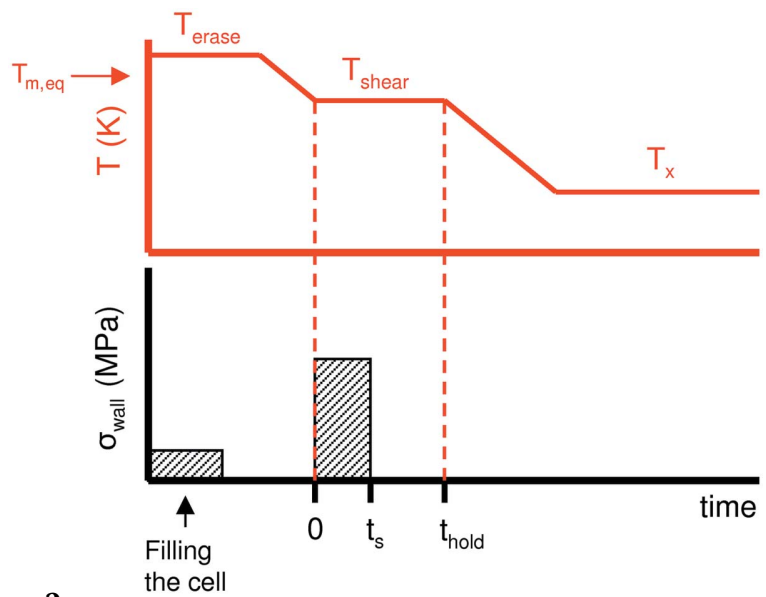

Figure 2

Example of experimental protocol for flow-induced crystallization. The cell is filled with polymer at a low wall shear stress and high temperature ( $T_{\text {erase }}$ ) above its equilibrium melting point. The temperature is kept at $T_{\text {erase }}$ for $5 \mathrm{~min}$ to erase any previous flow and thermal histories and then cooled to $T_{\text {shear }}$. When $T_{\text {shear }}$ is reached, a short high-shear pulse is imposed on the melt and data acquisition is started. After annealing at the shearing temperature for a time $t_{\text {hold }}$, the polymer is cooled to $T_{x}$ and the progress of crystallization is monitored. 
(a)

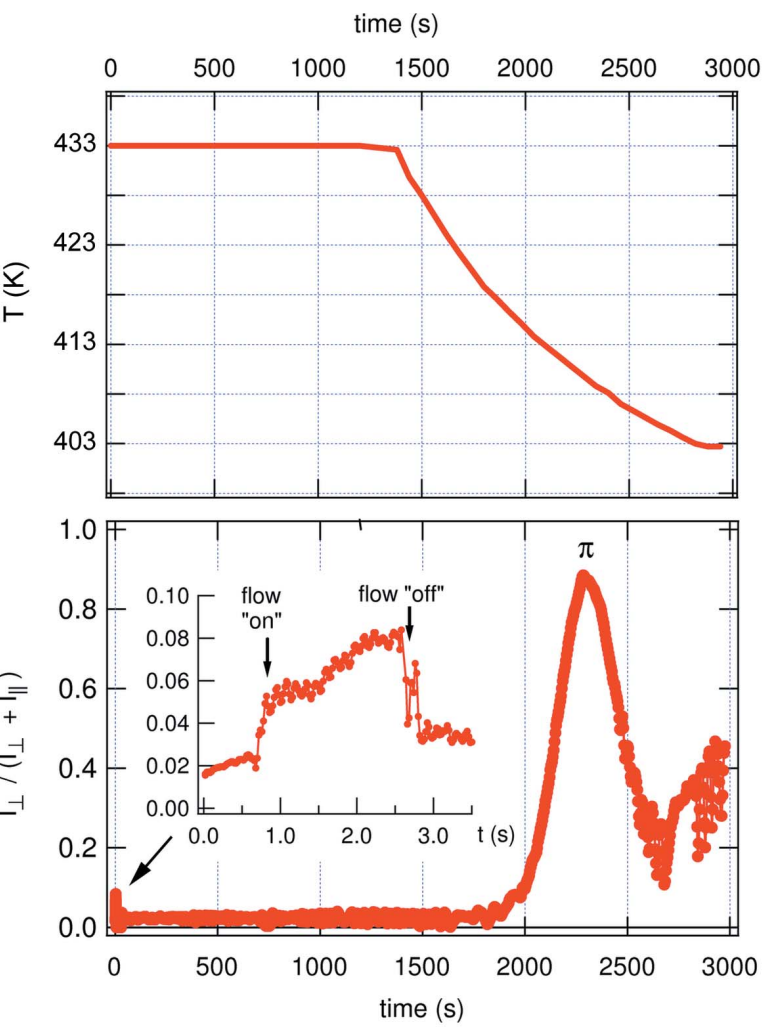

Figure 3

Recorded (a) temperature, $(b)$ transmitted visible light intensity through crossed polarizers, $(c)$ transmitted visible light intensity, $(d)$ diffracted intensity at selected wide angles in the equatorial plane and $(e)$ scattered intensity over a range of small angles in the meridional region for an experiment with shear at $0.093 \mathrm{MPa}$ for $2 \mathrm{~s}$ at $433 \mathrm{~K}$. The shearing temperature is held for $20 \mathrm{~min}(1200 \mathrm{~s})$, and then the polymer is cooled down to $403 \mathrm{~K}(a)$. During shear, only $I_{\perp} /\left(I_{\|}+I_{\perp}\right)$ detects structure formation [inset of $(b)$ ]. During the subsequent $1200 \mathrm{~s}$ at $433 \mathrm{~K}$, no evidence of structure was detected $(b)-(e)$. Upon cooling, growth of strongly oriented crystallites is evident $(e)$ that include both $\alpha$ - and $\gamma$ morphs $(d)$. Growth of oriented crystals produces strong birefringence $(b)$ and light scattering $(c)$. The inset in $(d)$ shows an equatorial 1D WAXD scattering pattern acquired at $t=2830 \mathrm{~s}$ where both $\alpha$ - and $\gamma$ phase crystalline peaks can be discerned. The area under the peak (after baseline subtraction) is calculated for each point in time. The inset in $(e)$ shows a typical 2D SAXS pattern for highly oriented lamellae. In the meridional region, the intensity enclosed in a rectangle is integrated and represents a measure of the evolution of oriented lamellae when plotted for each point in time.

$(<0.02 \mathrm{MPa}$ of wall shear stress) and at $488 \mathrm{~K}$ with isotactic polypropylene (iPP). The polymer was held for $5 \mathrm{~min}$ at $488 \mathrm{~K}$ ( $\left.T_{\text {erase }}\right)$, which is sufficient to erase any previous flow history (Seki et al., 2002) and allow the melt to fully relax. Then, the melt was cooled to a shearing temperature $\left(T_{\text {shear }}\right)$ of $433 \mathrm{~K}$. Once $T_{\text {shear }}$ was reached, a strong shearing pulse corresponding to a given wall shear stress $\left(\sigma_{\text {wall }}\right)$ was applied for a specified shearing time $\left(t_{\mathrm{s}}\right)$ of the order of seconds. The sample was held at $T_{\text {shear }}$ for a specific time $\left(t_{\text {hold }}\right)$ of $20 \mathrm{~min}$, and afterwards it was cooled down to a final crystallization temperature $\left(T_{\mathrm{x}}\right)$ of $403 \mathrm{~K}$. The transient temperatures were recorded.

The simultaneous optical and X-ray data were recorded from the moment that the shearing temperature was reached and the shearing pulse was imposed. During shear and shortly after, we acquired SAXS and WAXD frames with an acquisition time of $2 \mathrm{~s}$ and the optical signals were acquired every $20 \mathrm{~ms}$. While annealing at $T_{\text {shear }}$, the acquisition time for the (c)

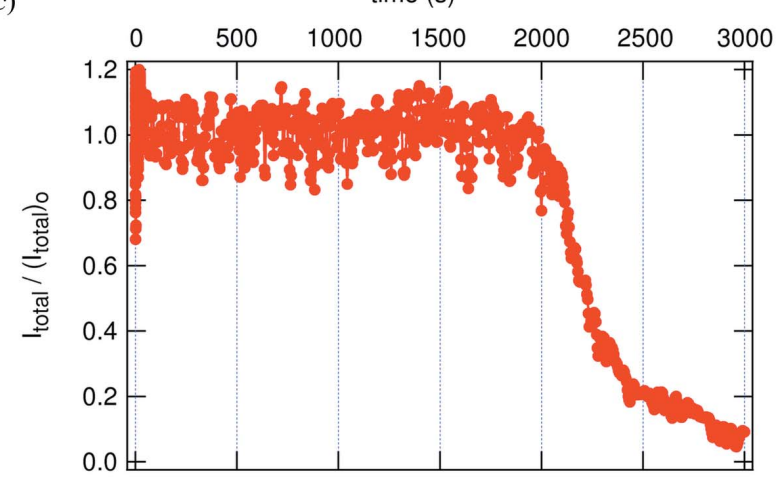

(d)

(e)
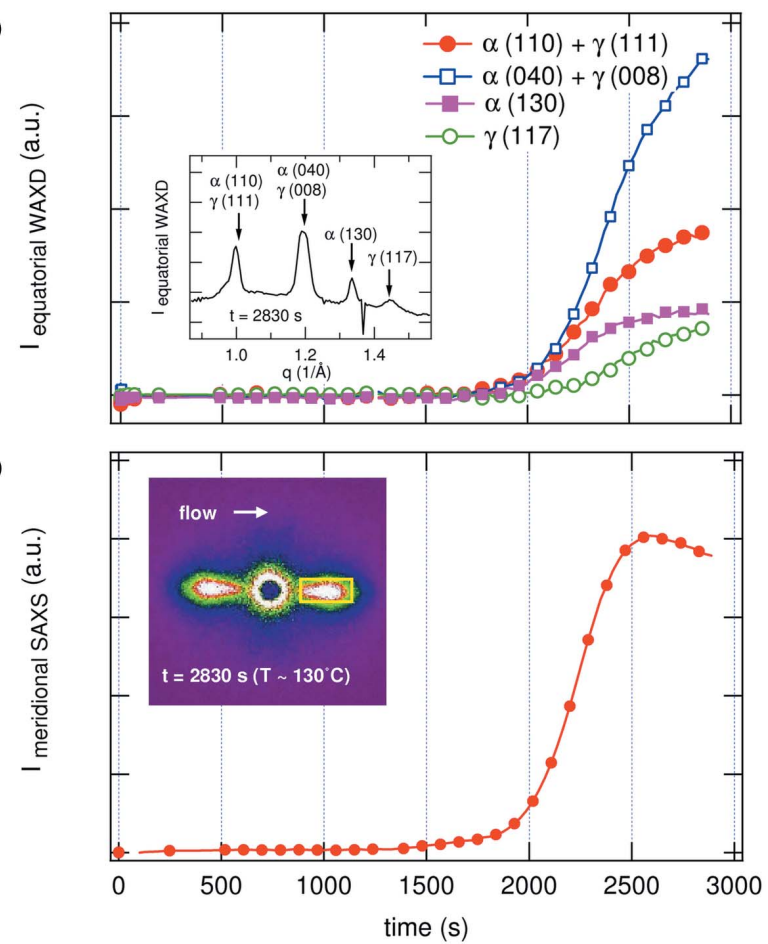

X-ray scattering patterns was progressively increased from $2 \mathrm{~s}$ to $120 \mathrm{~s}$ to improve the signal-to-noise ratio. The optical observables were recorded at $4 \mathrm{~s}$ intervals during $t_{\text {hold }}$ and thereafter. During cooling from $T_{\text {shear }}$ to $T_{\mathrm{x}}$ (which took $27 \mathrm{~min}$ ), a constant acquisition time of $30 \mathrm{~s}$ was used to acquire $\mathrm{X}$-ray patterns.

\section{Experimental results}

In order to test the experimental set-up, we used a bimodal mixture of isotactic polypropylenes prepared by solution blending in hot xylene. The sample contained $1 \%$ weight of an ultra-high-molecular-weight iPP $\left[M_{\mathrm{w}}=3500 \mathrm{~kg} \mathrm{~mol}^{-1}\right.$, polydispersity index $(\mathrm{PDI})=2.3]$ in a matrix of lower-molecularweight iPP $\left(M_{\mathrm{w}}=186 \mathrm{~kg} \mathrm{~mol}^{-1}\right.$, PDI $\left.=1.8\right)$.

The retardance, turbidity, equatorial WAXD and the meridional SAXS (Fig. 3) observed simultaneously during an experiment (for which $T_{\text {shear }}=433 \mathrm{~K}, \sigma_{\text {wall }}=0.093 \mathrm{MPa}, t_{\mathrm{s}}=$ 


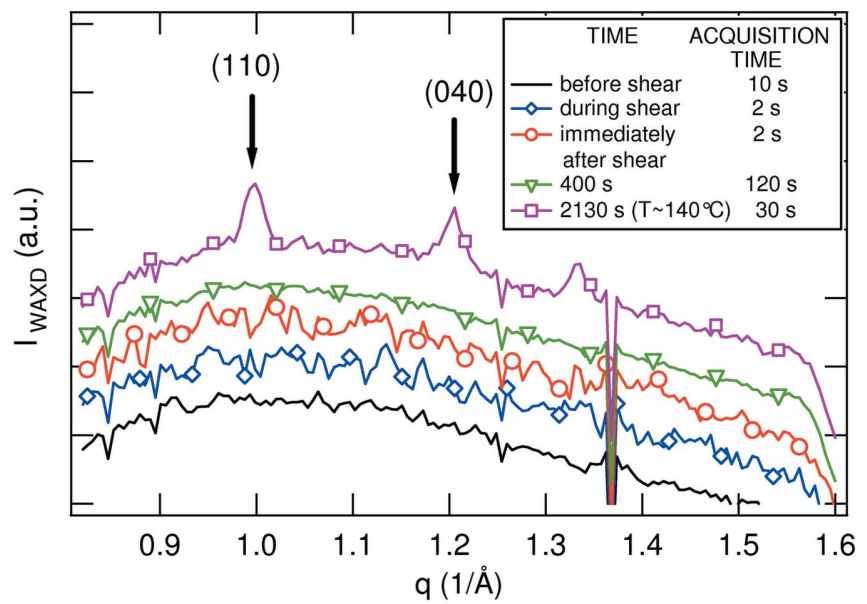

Figure 4

Equatorial 1D WAXD patterns at different times during the experiment. The $2 \mathrm{~s}$ frames acquired during shear and right after shear do not show any crystalline peaks and are essentially the same as the amorphous $10 \mathrm{~s}$ frame acquired before shear. A $120 \mathrm{~s}$ frame was acquired at time $400 \mathrm{~s}$ after flow (when the temperature is still $433 \mathrm{~K}$ ), and shows a better signalto-noise ratio but no evidence of crystalline peaks. Finally a pattern acquired after cooling has begun shows the location of the typical $\alpha$-phase crystalline peaks for isotactic polypropylene. There is a limited number of channels that do not follow Gaussian statistics, which are not taken into account for data analysis.

$2 \mathrm{~s}, t_{\text {hold }}=20 \mathrm{~min}$ and $T_{\mathrm{x}}=403 \mathrm{~K}$ ) illustrate that retardance $\delta$ is the only observable that reveals structure formation during flow for these conditions (inset of Fig. 3b). The flow-induced structures were so dilute that they could not be detected on the 1DWAXD (shown in Fig. 4) and 2D SAXS patterns acquired during shear. The growth velocity of crystallites at $433 \mathrm{~K}$ was negligible, and neither optical measurements nor $\mathrm{X}$-ray scattering were able to detect them during the $20 \mathrm{~min}$ holding time (even when using long acquisition times of $120 \mathrm{~s}$ for improved signal-to-noise ratio in the X-ray frames, Fig. 4). No equatorial streaks were observed in the SAXS patterns during shear nor while annealing at $433 \mathrm{~K}$. Then, as the temperature of the sample was reduced (Fig. $3 a$ ), the growth velocity of oriented crystallites increased. When the growth became evident, it was manifested as changes in the retardance, WAXD and SAXS measurements. The crystallites that formed were highly oriented, which would not be the case if the sample was subjected to the same experimental protocol but without applying the shear pulse. This indicates that some of the oriented precursors formed during shear had survived during the 20 minutes at $433 \mathrm{~K}$.

The optical anisotropy of the sample is manifested in the $I_{\perp} /\left(I_{\perp}+I_{\|}\right)$signal (Fig. $\left.3 b\right)$, related to the preferential orientation of polymer segments along the flow direction. When depolarization owing to light scattering is negligible, the measured signal $I_{\perp} /\left(I_{\perp}+I_{\|}\right)$and the retardance $\delta$ are related as follows (Macosko, 1994),

$$
\frac{I_{\perp}}{I_{\perp}+I_{\|}}=\sin ^{2}(\pi \Delta n d / \lambda)=\sin ^{2}(\delta / 2)
$$

where $\Delta n$ is the birefringence, $d$ is the length of the optical path through the sample, and $\lambda$ is the wavelength of the optical source. During the shear pulse of the melt, some polymer segments orient and the retardance of the sample shows an abrupt increase. Because oriented precursors were formed in these experiments, we observed the characteristic upturn (Kumaraswamy, Issaian \& Kornfield, 1999; Kumaraswamy et $a l ., 2002)$ on top of the retardance owing to melt flow (inset of Fig. 3b). Upon cessation of flow, the retardance drops (molten chains relax) and it remains low during $t_{\text {hold }}$ and during the early stages of cooling. Only when the temperature reaches $\sim 418 \mathrm{~K}$ does the retardance increase again. Then, the surviving oriented precursors act as templates for the growth of oriented crystallites in which the polymer chains are aligned preferentially along the flow direction. When this occurs, the retardance of the sample starts increasing, tracking the quantity of oriented crystallites that have formed. Since the relationship between the retardance $\delta$ and $I_{\perp} /\left(I_{\perp}+I_{\|}\right)$is sinusoidal (equation 1), $I_{\perp} /\left(I_{\perp}+I_{\|}\right)$can go over orders (observed as 'peaks' and 'valleys'). For example, the peak in $I_{\perp} /\left(I_{\perp}+I_{\|}\right)$at $t \simeq 2300 \mathrm{~s}$ corresponds to a retardance of $\pi$.

As the sample crystallizes, light scattering occurs which is sensitive to the size, shape and anisotropy of the crystallites. Therefore, the degree of turbidity (Fig. 3c) can be used to qualitatively monitor the progress of crystallization. It is calculated as the ratio of the total intensity at a given time and the initial total intensity,

$$
\text { Turbidity }=\frac{I_{\text {total }}}{\left(I_{\text {total }}\right)_{\mathrm{o}}}=\frac{I_{\perp}+I_{\|}}{\left(I_{\perp}+I_{\|}\right)_{\mathrm{o}}}
$$

Meaningful optical measurements are only possible when the transmitted intensity of the $\mathrm{HeNe}$ laser beam through the sample is adequate (time $<2500 \mathrm{~s}$ in this experiment). Additional information can still be obtained from the WAXD and SAXS patterns after the sample becomes too turbid for optical measurements.

WAXD provides information on the length scale of the crystalline unit cell. The inset of Fig. 3(d) shows a typical 1D WAXD pattern at time $=2830 \mathrm{~s}(\sim 403 \mathrm{~K})$. WAXD allows us to identify the crystal morphs that form and their rate of growth. Multiple crystal morphs can occur in iPP $(\alpha, \beta$ or $\gamma)$ (Lotz et al., 1996). In our experiment the first peaks to emerge (Fig. 4, time $=2130 \mathrm{~s}$ ) are those of the $\alpha$ crystal morph (inset Fig. $3 d$ ), which can be indexed as (110), (040) and (130). However, in the late stages, a peak unique to the $\gamma$ form develops [inset Fig. 3(d), indexed as (117)], indicating that a corresponding (small) contribution of $\gamma$ peaks (which coincide in their angular position) to the first two $\alpha$ peaks must be present. The orientation of the 1D WAXD detector along the equatorial direction is chosen to monitor the growth of $\alpha$ and $\gamma$ crystallites with their chain axis along the flow. We quantify the growth of each WAXD reflection by subtracting the baseline (along with the amorphous halo) and integrating the area of each peak (individual curves in Fig. $3 d$ ).

Finally, SAXS yields information on larger length scales than WAXD (of the order of the lamellar long period $L$ ). The inset of Fig. 3(e) illustrates a 2D SAXS pattern acquired at time $=2830 \mathrm{~s}(\sim 403 \mathrm{~K})$ which not only shows that crystal- 
lization has occurred but also that it has led to a highly oriented morphology (as can be deduced from the anisotropy of the pattern). The meridional scattering lobes arise from stacks of lamellae that have been nucleated from the oriented precursors that survived the holding time at $433 \mathrm{~K}$, and thus grow perpendicular to the flow direction. The time evolution of the integrated intensity scattered in the meridional region (Fig. 3e) provides a measure of the kinetics of development of oriented lamellae. This meridional integrated SAXS intensity slightly decreases after $\sim 2500 \mathrm{~s}$, even though the WAXD signal (Fig. $3 d$ ) is still increasing. This indicates that at least some of the unit cells that are forming act to decrease the electron-density contrast in the lamellar stacks already present. Further methods of analysis can be used to quantify the development of oriented and unoriented lamellae with time, the orientation distribution, and the lamellar long period (Ryan et al., 1997; Kumaraswamy et al., 2004). However, detailed inspection of these parameters is beyond the scope of the present paper.

\section{Conclusions}

We have demonstrated an experimental configuration to combine the powerful capabilities of a short-term shearing apparatus with simultaneous optical and X-ray scattering techniques (WAXD and SAXS) that connect the earliest events that occur during shear-induced polymer crystallization with the subsequent kinetics and morphology development. The sensitivity of rheo-optics reveals dilute shear-induced precursors that form during flow of isotactic polypropylene even when X-ray techniques are unable to detect them. Then, $\mathrm{X}$-ray scattering patterns are used to quantify the ensuing kinetics of development of oriented crystallites and their orientation distribution, demonstrated here with 1D WAXD2D SAXS and amenable to simultaneous 2DWAXD and 2D SAXS. WAXD identifies and quantifies the crystalline phases that form. Established methods of analyzing scattering patterns can be applied to extract important parameters (e.g. the lamellar long period versus time from SAXS, ratio of oriented and unoriented crystallites, orientation distribution). This simultaneous combination of techniques allows unequivocal correlation between the events that occur during shear and the evolution of crystallization after cessation of flow in spite of the extreme sensitivity of flow-induced crystallization to small changes in the imposed stress and the material.

This work was funded by the National Science Foundation under awards DMR-0080065 and DMR-0505393. The authors are grateful to Dr Jan Verhoeven (Amolf) for providing the Al-coated mica mirrors. We acknowledge the European Synchrotron Radiation Facility and the Netherlands Organization for Scientific Research for provision of synchrotron radiation facilities, and all DUBBLE staff for assistance during experiments. Mr B. Vrolijk of Element 6 is thanked for advice on how to mount the diamond windows. FA and FJBC wish to acknowledge the 'Ministerio de Educación y Ciencia' (grant FIS2004-01331), Spain, for financial support. LFB wishes to acknowledge the 'la Caixa' fellowship program.

\section{References}

Andersen, P. G. \& Carr, S. H. (1978). Polym. Eng. Sci. 18, 215-221. Azzurri, F. \& Alfonso, G. C. (2005). Macromolecules, 38, 1723-1728.

Baert, J. \& Van Puyvelde, P. (2006). Polymer, 47, 5871-5879.

Bras, W., Dolbnya, I. P., Detollenaere, D., van Tol, R., Malfois, M., Greaves, G. N., Ryan, A. J. \& Heeley, E. (2003). J. Appl. Cryst. 36, 791-794.

Bras, W. \& Ryan, A. J. (1998). Adv. Colloid Interface Sci. 75, 1-43.

Chu, B. \& Hsiao, B. S. (2001). Chem. Rev. 101, 1727-1761.

Devaux, N., Monasse, B., Haudin, J. M., Moldenaers, P. \& Vermant, J. (2004). Rheol. Acta, 43, 210-222.

Dolbnya, I. P., Alberda, H., Hartjes, F. G., Udo, F., Bakker, R. E., Konijnenburg, M., Homan, E., Cerjak, I., Goedtkindt, P. \& Bras, W. (2002). Rev. Sci. Instrum. 73, 3754-3758.

Fujiyama, M., Wakino, T. \& Kawasaki, Y. (1988). J. Appl. Polym. Sci. 35, 29-49.

Gleeson, H. F. (1995). Rev. Sci. Instrum. 66, 3563-3568.

Haas, T. W. \& Maxwell, B. (1969). Polym. Eng. Sci. 9, 225-241.

Heeley, E. L., Fernyhough, C. M., Graham, R. S., Olmsted, P. D., Inkson, N. J., Embery, J., Groves, D. J., McLeish, T. C. B., Morgovan, A. C., Meneau, F., Bras, W. \& Ryan, A. J. (2006). Macromolecules, 39, 5058-5071.

Kantz, M. R., Stigale, F. H. \& Newman, H. D. (1972). J. Appl. Polym. Sci. 16, 1249-1260.

Koscher, E. \& Fulchiron, R. (2002). Polymer, 43, 6931-6942.

Kumaraswamy, G., Issaian, A. M. \& Kornfield, J. A. (1999). Macromolecules, 32, 7537-7547.

Kumaraswamy, G., Kornfield, J. A., Yeh, F. J. \& Hsiao, B. S. (2002). Macromolecules, 35, 1762-1769.

Kumaraswamy, G., Verma, R. K. \& Kornfield, J. A. (1999). Rev. Sci. Instrum. 70, 2097-2104.

Kumaraswamy, G., Verma, R. K., Kornfield, J. A., Yeh, F. J. \& Hsiao, B. S. (2004). Macromolecules, 37, 9005-9017.

Langouche, F. (2006). Macromolecules, 39, 2568-2573.

Liedauer, S., Eder, G., Janeschitzkriegl, H., Jerschow, P., Geymayer, W. \& Ingolic, E. (1993). Int. Polym. Proc. 8, 236-244.

Lotz, B., Wittmann, J. C. \& Lovinger, A. J. (1996). Polymer, 37, 49794992.

Macosko, C. W. (1994). Rheology: Principles, Measurements, and Applications. New York: VCH.

Pogodina, N. V., Winter, H. H. \& Srinivas, S. (1999). J. Polym. Sci. B, 37, 3512-3519.

Ran, S. F., Fang, D. F., Sics, I., Toki, S., Hsiao, B. S. \& Chu, B. (2003). Rev. Sci. Instrum. 74, 3087-3092.

Ryan, A. J., Stanford, J. L., Bras, W. \& Nye, T. M. W. (1997). Polymer, 38, 759-768.

Seki, M., Thurman, D. W., Oberhauser, J. P. \& Kornfield, J. A. (2002). Macromolecules, 35, 2583-2594.

Somani, R. H., Hsiao, B. S., Nogales, A., Fruitwala, H., Srinivas, S. \& Tsou, A. H. (2001). Macromolecules, 34, 5902-5909.

Trotignon, J. P. \& Verdu, J. (1987). J. Appl. Polym. Sci. 34, 1-18.

Vleeshouwers, S. \& Meijer, H. E. H. (1996). Rheol. Acta, 35, 391-399.

Wutz, C., Bark, M., Cronauer, J., Dohrmann, R. \& Zachmann, H. G. (1995). Rev. Sci. Instrum. 66, 1303-1307.

Zhukov, V., Udo, F., Marchena, O., Hartjes, F. G., vandenBerg, F. D., Bras, W. \& Vlieg, E. (1997). Nucl. Instrum. Methods Phys. Res. A, 392, 83-88. 